\title{
Synthesis and formation of biologically active structures of functionally substituted diazocrown ethers containing nanoparticles
}

\begin{abstract}
New compounds based on diazacrown ether - macrocycle (MC) containing ultradispersed iron particles were synthesized. It was found that the synthesized compounds were effective against gram-negative microorganisms Escherichia coli, Klebsiella spp. and gram-positive Staphylococcus aureus, having multi drug resistance properties.
\end{abstract}

Keywords: crown ether, macro cycles, siderophores, nanoparticles
Volume 5 Issue 2 - 2019

Leyla Vezirova

Azerbaijan State University of Oil and Industry, Azerbaijan

Correspondence: Leyla Vezirova, Azerbaijan State University of Oil and Industry, RI Geotechnological Problems of Oil, Gas and Chemistry,AzI0I0 Baku,Azadlig av. 20,Azerbaijan, Email L_vez@mail.ru

Received: February 10, 2018 | Published: April 04, 2019

\section{Introduction}

Supramolecular structures play a vital role in functioning of living organism. Their feature is that they arise spontaneously, via hydrophobic interactions, electrostatic effects that in turn arise from the interaction of functional groups of biomolecules with an aqueous environment and through non-covalent bonds. Synthetic analogues of such systems are of great interest. They imitate the properties of naturally occurring systems. The crown ethers can demonstrate the properties of naturally occurring membrane active antibiotics. Macro cycle cavity of crown ether binds the cations trough iondipole and dipole-dipole non-covalent bonds. ${ }^{1-4}$ The aim of this work is to functionalize iron nanoparticle with hydroxyl substituted diazacrown ether which is able to mimic natural siderophores. Synthesis of macro cycle 1,13-Diaza-5,9-dioxa-7-hydroxy-3,4:10,1dibenzocyclopentadecan was carried out according to the method. Synthesis of nanostructures was carried out by functionalization of $\mathrm{Fe}_{3} \mathrm{O}_{4}$ with $\mathrm{MC}\left(\mathrm{MC} @ \mathrm{Fe}_{3} \mathrm{O}_{4} \mathrm{NPs}\right)$. Magnetic iron oxide nanoparticles are prepared by wet chemical precipitation from aqueous iron salt solutions in alkaline milieu, created by using $\mathrm{NH} 4 \mathrm{OH}$, in the atmosphere of gaseous nitrogen. ${ }^{5}$

The formed $\mathrm{Fe}_{3} \mathrm{O}_{4}$ nanoparticles (NPs) were separated by NdFeB permanent magnet, repeatedly washed with distilled water and dispersed in ethanol. The ethanol solution of MC, taken in excess, was added to ethanol solution of $\mathrm{Fe}_{3} \mathrm{O}_{4}$ nanoparticles and thoroughly stirred. After stirring during 8hours in ambient conditions, the prepared nanostructures were separated by strong $\mathrm{NdFeB}$ permanent magnet and repeatedly washed with distilled water. The obtained NPs were dried in ambient conditions and the iron content in the samples was analyzed by atom absorption spectroscopy performed on Varian Spectra 220FS Atomic absorption spectrometer. Samples were prepared by Milestone ETHOS 1 Microwave extraction unit. The UV spectra were recorded on Spectrophotometer Secord 250 Plus. UV spectra were recorded at the $275 \mathrm{~nm}$ range for standard solutions of MC.

Antibacterial activity of $\mathrm{MC}$ andMC@ $@ \mathrm{Fe}_{3} \mathrm{O}_{4}$ was tested by diffusion method, performed on Petri dishes, on Staphylococcus aureus, Klebsiella spp. and Escherichia coli ${ }^{6}$ The synthesized substances were taken in amount equal to $30 \mu \mathrm{g}$. Escherichia coli were cultivated on Endo's medium, Klebsiella spp. on Sabouraud medium and Staphylococcus aureus on Baird-Parker agar (cultures were kindly provided by one of the clinical laboratories of Baku, Azerbaijan). Due to the fact that this method provides only quality data, micro dilution method was also performed. By this method the MIC of prepared substances on Staphylococcus aureus, Klebsiella spp. and Escherichia coli was identified. To perform micro dilution method the stock solutions of different concentrations of the substances were prepared in distilled sterile water and were distributed in 96 multi-well plates. Each well was inoculated with $0.1 \mathrm{~mL}$ of microbial suspensions of $0.5 \mathrm{McF}$ arland turbidity, prepared from $24 \mathrm{~h}$ fresh culture. Sterility control wells and microbial growth controls were involved. The plates were incubated for $2 \mathrm{~h}$ at $37^{\circ} \mathrm{C}$. In order to measure antibacterial effect on bio film development, wells were washed with PBS three times, dried for 15 sminutes in room temperature and stained by crystal violet $1 \%$ for 15 minutes. After the deletion of crystal violet $1 \%$, wells again were washed 3 times with PBS and dried at room temperature for 15 minutes. Further, the bio film, formed on the plastic wells, was re-suspended in $30 \%$ acetic acid and the intensity of the colored suspension was assayed by measuring the absorbance at 590nm. ${ }^{7}$ Synthesis of MC 1,13-Diaza-5,9-dioxa-7-hydroxy3,4:10,1-dibenzocyclopentadecan (4) is depicted on the scheme 1 . $\mathrm{N}, \mathrm{N}$ ' ethylenebis(salysilimine) (2) was prepared by condensation of salysilaldehide (1) with ethylenediamine. The reduction of (2) was carried out by sodium borohydride. The ring closure step was carried out by reaction of corresponding saturated derivative (3) with 1,3-dichloro-2-propanol.

The purity and crystalline properties of the $\mathrm{MC} @ \mathrm{Fe}_{3} \mathrm{O}_{4}$ were investigated by powder X-ray diffraction (XRD). All the XRD peaks were well defined and corresponded to $\mathrm{Fe}_{3} \mathrm{O}_{4}$ nanoparticles with cubic structure. XRD peak broadening is the evidence of nanocrystal formation. All of the lines in the pattern relate to magnetite and can be indexed using the ICDD (PDF-2/Release 2011 RDB) DB card number 00-001-1111, for prepared nanostructure. The pattern of MC@ $\mathrm{Fe}_{3} \mathrm{O}_{4}$ NPs has characteristic peaks at 30.250 (220), 35.750 (311), 43.520(400), 57.440(511), 62.930(440). The synthesized MC would be able to reveal antimicrobial activity (bacteria Staphylococcus, Escherichia coli and Klebsiella spp.), due to ionoforic properties as well as $\mathrm{MC} @ \mathrm{Fe}_{3} \mathrm{O}_{4}$ nanostructures can mimic the properties of natural siderofores, especially in case of gram-negative microorganisms. 


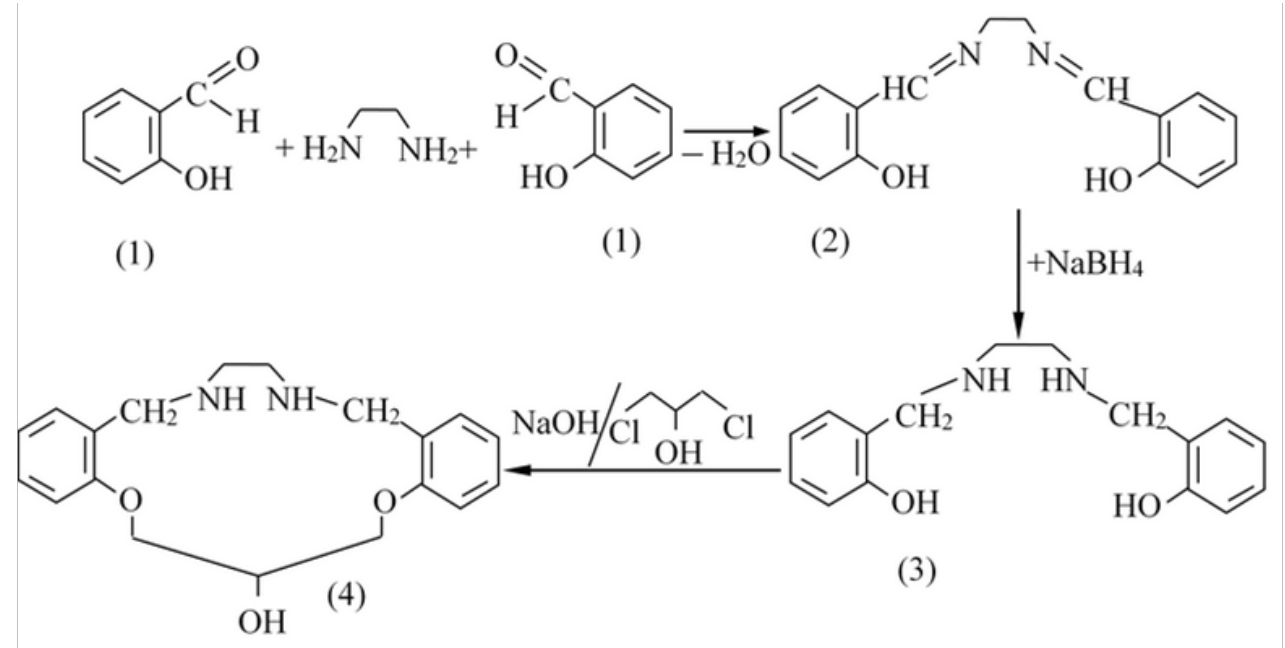

Figure I Scheme. The synthesis of MC I,13-Diaza-5,9-dioxa-7-hydroxy-3,4:10, I dibenzocyclopentadecan.

\section{Results}

The results of the micro diffusion method show that the inhibition zone, produced by MC on Staphylococcus aureus, was $14 \mathrm{~mm}$ in diameter, but there was no effect on Escherichia coli and Klebsiella spp. However, when it comes to $\mathrm{MC} @ \mathrm{Fe}_{3} \mathrm{O}_{4}$, the contrary results were observed. It has no effect on Staphylococcus aureus, but has produced an inhibition zone with the diameter $28 \mathrm{~mm}$ on Escherichia coli and $22 \mathrm{~mm}$ on Klebsiella spp. The antibacterial effect of MC on Staphylococcus aureus can be explained by the fact that it acts like ionophore, disrupting the membrane's potential of bacteria. At the same time, in case of $\mathrm{MC} @ \mathrm{Fe}_{3} \mathrm{O}_{4}$, antibacterial effect is absent, probably, because $\mathrm{Fe}_{3} \mathrm{O}_{4}$ NPs blocked action sites of crown ethers, responsible for ionophore binding with membrane via $\mathrm{OH}, \mathrm{NH}$ and MC' oxygen atoms by means of ion-dipole, dipole-dipole and etc. interactions.

When it comes to Escherichia coli and Klebsiella spp., the mechanism is different. As gram-negative bacteria form so called siderophoric system that can acquire iron from the environment by secreting special iron-chelating agents - siderophores. These siderophores penetrate to periplasmic space through responsible for iron uptake porin channels. Hydroxyl substituted diazacrown ether is similar to siderophores in their ability to chelate iron. MC alone cannot penetrate through the thick membrane of gram-negative bacteria and produce antibacterial effect. However, laden with iron, it plays a role as a siderophore and is more advantageous for bacterial cells than natural free-from-iron siderophore. The sum of all abovementioned factors contributes to high antimicrobial effect of MC@ $\mathrm{Fe}_{3} \mathrm{O}_{4}$ on gram-negative bacteria. I would like to thank prof. Ulviya A. Gasanova for her support in the course of my scientific work.

\section{Conclusion}

Our research's results show that synthesized hydroxyl substituted diazacrown shows a significant antibacterial effect and low MIC on gram-positive Staphylococcus aureus, whereas nanostructured hydroxyl substituted azacrown produces an effect and low MIC on gram-negative microorganism Klebsiella spp. and Escherichia coli, and even has ability to inhibit their growth in bio film in all concentrations.

\section{Acknowledgments}

None.

\section{Conflicts of interest}

Author declares that there is no conflict of interest.

\section{References}

1. Steed JW, Atwood JL. Supramolecular chemistry. 2nd edn. John Wiley \& Sons, Ltd; 2009:27-38.

2. Peter JC. Supramolecular Chemistry: From Biological Inspiration to Biomedical Applications. Springer.

3. Liu L, Chen S. Theoretical Study on Cyclopeptides as the Nanocarriers for $\mathrm{Li}^{+}, \mathrm{Na}^{+}, \mathrm{K}^{+}$and $\mathrm{F}^{-}, \mathrm{Cl}^{-}, \mathrm{Br}^{-}$. Journal of Nanomaterials. 2015.

4. Chris JJ, Thornback JR. Medicinal Applications of Coordination Chemistry. The Royal Society of Chemistry. 2007;4:203-205.

5. Massart R. Preparation of Aqueous Magnetic Liquids in Alkaline and Acidic Media. IEEE Transactions on Magnetics. 1981;17: 1247-1248.

6. Mayrhofer S, Domig KJ, Mair C, et al. Comparison of Broth Microdilution, E test, and Agar Disk Diffusion Methods for Antimicrobial Susceptibility Testing of Lactobacillus Acidophilus Group Members. Applied and Environmental Microbiology. 2008;12: 3745-3748.

7. Hasanova UA, Ramazanov MA, Maharramov AM, et al. Nano-Coupling of Cephalosporin Antibiotic with $\mathrm{Fe}_{3} \mathrm{O}_{4}$ Nanoparticles: Trojan Horse Approach in Antimicrobial Chemotherapy of Infections Caused by Klebsiella spp. Journal of Biomaterials and Nanobiotechnology. $2015 ; 6: 225-235$. 